\title{
The Revised 2010 EMA Guideline for the Investigation of Bioequivalence for Immediate Release Oral Formulations with Systemic Action
}

\author{
Roger K. Verbeeck ${ }^{1,2}$ and Flora T. Musuamba ${ }^{1}$ \\ ${ }^{1}$ Louvain Drug Research Institute, Pharmacokinetics/Drug Metabolism, Catholic University of Louvain, Brussels, \\ Belgium \\ ${ }^{2}$ Faculty of Pharmacy, Rhodes University, Grahamstown, South Africa
}

Received April 4, 2012; Revised, May 27, 2012; Accepted, May 29, 2012; Published May 31, 2012.

\begin{abstract}
On August 1, 2010, a revised guidance regarding bioequivalence (BE) assessment for the approval of innovator (bridging studies, variations, line extensions) and generic medicinal products in the EU came into effect (EMA Guideline on the Investigation of Bioequivalence, CPMP/EWP/QWP/1401/98 Rev. 1/Corr**, London, 20 January 2010). This guideline specifies the requirements for BE assessment for immediate release oral dosage forms with systemic action. Compared to the previous BE guideline of the EMA, clearer guidance is now given on several topics including BE assessment of highly variable drugs/drug products (HVDs/HVDPs), the use of metabolite data, acceptance criteria for narrow therapeutic index drugs (NTIDs), BCS-based biowaivers, and dose strength to be used in case of application for marketing authorization of several strengths. However, the health authorities of the various EU member states do not necessarily apply the same rules as far as substitution and switchability between medicinal products are concerned. Moreover, differences still exist between the $\mathrm{BE}$ guidelines of the major health authorities (FDA, EMA, NIHC, ...) on topics such as HVDs/HVDPs, NTIDs and BCS-based biowaivers. Global harmonization should be the next logical step to guarantee accessibility to safe and efficacious drug products for patients in all parts of the world.
\end{abstract}

This article is open to POST-PUBLICATION REVIEW. Registered readers (see "For Readers") may comment by clicking on ABSTRACT on the issue's contents page.

\section{INTRODUCTION}

The concept of bioequivalence and the methodology to assess bioequivalence have evolved over the past three to four decades $(1,2)$. The first "European" bioequivalence guidelines were published in 1991 by the Commission of the European Communities in an attempt to harmonise the marketing approval (registration) of generic drug products in the various member states of the European Community (EC), which became known as the European Union (EU) following the Treaty of Maastricht in 1993 (3). Until the publication of this first European Note for Guidance related to bioavailability/bioequivalence assessment, generic drug products were exclusively registered by the national authorities of the EU member states. Prior to 1991, the registration dossiers were not comprehensive and assessments were based on principles published in the scientific literature, FDA guidelines, and the first European guidelines on pharmacokinetic studies in man (4). In 1995 the European Agency for the Evaluation of Medicinal Products or EMEA - since the end of 2009 known as the European Medicines Agency (EMA) - a decentralized body of the EU with headquarters in London, was established. Its main responsibility is the protection and promotion of public and animal health through the evaluation and supervision of medicines for human and veterinary use. In 2001, a first revision of the Note for Guidance on the Investigation of Bioavailability and Bioequivalence was published by the EMA Committee of Proprietary Medicinal Products (CPMP) (5). These guidelines, which only dealt with immediate release oral medicinal products with systemic action, came into effect on January 2002. However, as the number of applications for marketing approval of generic medicinal products via the European procedures, the Mutual Recognition Procedure/Decentralized Procedure and Centralized Procedure, increased, it became clear that the assessors of the various

Corresponding Author: Prof. Roger K. Verbeeck; UCL/LDRI/PKDM B1.73.13; Av. E. Mounier 73; 1200 Brussels; Belgium; email: roger.verbeeck@uclouvain.be 
EU member states frequently did not agree with the interpretation of these bioavailability/bioequivalence guidelines. More difficult bioequivalence issues such as Biopharmaceutics Classification System (BCS)based biowaivers, the use of metabolite instead of parent drug plasma concentrations, bioequivalence assessment of highly variable drugs, for example, were not well described in the 2001 Note for Guidance. In May 2007, the EMA published two concept papers, one expressing the need for revision of the 2001 Note for Guidance, and the other concept paper announced the preparation of a document to clarify the regulatory requirements to support an optimal and harmonised use of the BCS-based biowaiver approach (6-8).

On August 1, 2010, the revised EMA Guideline on the Investigation of Bioequivalence came into effect (9). This guideline is limited to bioequivalence issues as they relate to immediate release oral dosage forms with systemic action. In Appendix III of this new guideline, the BCSbased biowaiver approach is now explained in more detail. In this commentary, some important issues on bioequivalence assessment are briefly presented with special emphasis on the changes in the revised EMA Guideline on the Investigation of Bioequivalence. It should be noted that the EMA also publishes a "Questions \& Answers" document which is up-dated on a regular basis (10). For a correct interpretation of the revised EMA bioequivalence guidelines it is important to also read this Question \& Answer document which is referred to in this commentary.

\section{LEGAL BASIS AND SCOPE}

The 2010 EMA Guideline on the Investigation of Bioequivalence (9) refers to Directive 2001/83/EC, as amended, as the legal basis for Marketing Authorisation Applications for human medicinal products submitted under Art. 10(1) (generic applications), Art. 10b (fixed combination), Art. 10(3) (hybrid applications), and Art. 8(3) (full applications). It also applies to extension and variation applications in accordance with European Commission Regulations.

Although the 2010 EMA guideline concerns immediate release (IR) oral formulations with systemic action, some general recommendations on the bioequivalence requirements for specific IR formulations as well as for other types of formulations are briefly discussed in Appendix II, e.g. oral dispersible tablets, oral solutions, parenteral solutions, special dosage forms (liposomal, micellar, emulsion) for intravenous use and locally acting products. Bioequivalence requirements for fixed combination dosage forms are covered in the "Guideline on Clinical Development of Fixed Combination Medicinal products" (11). Recommendations for bioequivalence studies on modified release oral medicinal products and transdermal dosage forms are described in a specific guideline which is currently under revision $(12,13)$.

\section{STUDY DESIGN AND STUDY SUBJECTS}

As far as study design is concerned, no major changes have been made in the 2010 EMA guideline. In general, when comparing the bioavailability of a test $(\mathrm{T})$ formulation to that of a reference (R) formulation, a randomised, twoperiod, two-sequence single dose crossover design is recommended. A parallel design may exceptionally be used in case of long half-life drugs. The use of replicate crossover designs is recommended to assess the bioequivalence of highly variable drugs/drug products and will be discussed in more detail later since the 2010 EMA guideline has new recommendations regarding bioequivalence assessment of highly variable drugs necessitating the use of a replicate design.

Conduct of a multiple dose study in healthy volunteers to assess bioequivalence of an IR release oral formulation is, according to the 2010 EMA guideline, only justified on the basis of insufficient sensitivity of the bioanalytical method. However, this justification will only be accepted in rare cases because due to recent developments in bioanalytical methodology it is highly unlikely that the parent drug concentration in plasma cannot be measured accurately and precisely. Carrying out a multiple dose bioequivalence study for a medicinal product showing high intra-individual variability in drug plasma concentrations, which was considered an option according to the 2001 Note for Guidance, is no longer acceptable. Although bioequivalence studies should normally be carried out in healthy volunteers, it may be necessary, for tolerability reasons, to use patients instead. Since a single dose bioequivalence study in patients may not be feasible, conduct of a multiple dose study in patients is acceptable.

Compared to the 2001 Note for Guidance, the 2010 EMA guideline does not introduce any new recommendations regarding the selection of healthy study subjects. However, in the revised guidelines it is now recommended that subjects 
should be 18 years or older (previously, between 18 - 55 years) and preferably have a Body Mass Index between 18.5 and $30 \mathrm{~kg} / \mathrm{m}^{2}$ (previously, within the normal range). In the 2010 EMA guideline the use of healthy volunteers to assess bioequivalence is now justified because this approach is considered adequate to allow extrapolation of the results to patient populations for which the reference medicinal product is approved.

In the 2010 EMA guideline it is also clearly stated that "all treated subjects should be included in the analysis".

\section{STUDY CONDUCT}

The drug products should normally be administered after an overnight fast and no food is allowed for at least 4 hours post-dose (fasting study). However, in the case where the Summary of Product Characteristics of the reference formulation (originator) recommends its intake with food, the study should be carried out under fed conditions. The 2010 EMA guideline now provides more information on how to carry out a bioequivalence study under fed conditions. Since food composition (fat content) and timing of the meal relative to medicinal product ingestion are crucial for the assessment of oral drug bioavailability, the revised guideline recommends, where a fed bioequivalence study is carried out, administration of the test and reference preparation immediately after completing a high fat meal which represents the "worst case scenario". The exact composition of a high fat meal is also now described in detail. Unlike the FDA, which generally recommends a fasting and fed study for immediate release oral drug products (14), the EMA requires bioequivalence studies under both fasted and fed conditions only in exceptional cases, i.e. for products with specific formulation characteristics such as microemulsions and solid dispersions and only if these products can be taken with or without a meal.

Some regulatory agencies (e.g. Health Canada, the National Institute of Health Sciences of Japan), as well as the World Health Organization, permit so-called "add-on" (or "twostage") designs (15-18). With these designs, if the failure to declare the two formulations bioequivalent appears to be due to insufficient power, i.e. an insufficient number of subjects included in the study to show bioequivalence between two bioequivalent drug products, it is permitted to carry out an additional study on a number of subjects and the results from both trials can then be combined in a final analysis. When using such a "two-stage" or "add-on" design, appropriate steps must be taken to correct for multiplicity and, therefore, guarantee an overall type I error of 5\%, i.e. $\alpha=0.05$ (19-21). The 2010 EMA Guideline on the Investigation of Bioequivalence also allows such a "two-stage" design. One possible approach mentioned in this revised EMA bioequivalence guideline is calculating the $94.12 \%$ confidence interval, instead of the usual $90 \%$ confidence interval, corresponding to an adjusted $\alpha$ of 0.0294 , for both the analysis of the stage 1 results as well as the combined results from stage 1 and stage 2 (20).

Also new in the 2010 EMA guideline is the requirement to list, in Module 2.7.1 of the Common Technical Document (22), all relevant studies carried out with the product for which marketing authorisation is applied, i.e. bioequivalence studies comparing the test product (same composition and manufacturing process) with a reference medicinal product marketed in the EU. Full study reports should be provided for all bioequivalence studies, except pilot studies for which study synopses are sufficient.

\section{BIOEQUIVALENCE METRICS}

In a BE study the following pharmacokinetic parameters should be determined: the area under the plasma concentration-time curve from 0 to $t$, i.e. to the time of the last quantifiable plasma concentration $\left(\mathrm{AUC}_{0-\mathrm{t}}\right)$, the area under the plasma concentration-time curve from 0 to infinity $\left(A U C_{0-\infty}\right)$, the residual area $\left(\mathrm{AUC}_{\mathrm{t}-\infty}\right)$, the maximum plasma concentration $\left(\mathrm{C}_{\max }\right)$ and the time at which $\mathrm{C}_{\max }$ was observed $\left(\mathrm{t}_{\max }\right)$. The terminal plasma elimination rate constant $\left(\lambda_{z}\right)$ and the corresponding plasma half-life $\left(t_{1 / 2}\right)$ also need to be determined in case the AUC has to be extrapolated to infinity. The sampling schedule should follow the plasma concentration-time curve long enough to ensure that $\mathrm{AUC}_{0-\mathrm{t}}$ covers at least $80 \%$ of $\mathrm{AUC}_{0-\infty}$, hence the requirement to also determine $\mathrm{AUC}_{\mathrm{t}-\infty}$ and $\mathrm{AUC}_{0-\infty}$. However, it is not necessary to extend blood sampling beyond 72 hours following administration of test and reference formulation, because for an IR formulation the oral absorption process has presumably been covered by 72 hours. In that case, AUC truncated at 72 hours $\left(\mathrm{AUC}_{0-72 \mathrm{~h}}\right)$ should be estimated and $\mathrm{AUC}_{0-\infty}$ and $\mathrm{AUC}_{\mathrm{t}-\infty}$ do not need to be calculated. In the 2001 Note for Guidance it was stated that, for drugs with a long 
half-life, relative bioavailability can be adequately estimated as long as the total collection period is justified. According to the 2010 EMA guideline, AUC truncated at 72 hours may be used for BE assessment irrespective of the half-life of the drug.

Although the 2010 EMA guideline accepts the use of urinary excretion data to determine the extent of absorption in case it is not possible to reliably measure the plasma concentration-time profile of the parent compound, its use to determine peak exposure should be carefully justified. Whereas the 2001 Note for Guidance considered the possibility to use pharmacodynamic effect measurements to assess bioequivalence, this is no longer mentioned in the 2010 guideline.

\section{PARENT COMPOUND VERSUS METABOLITES}

The revised 2010 guideline is much clearer on the use of metabolites in bioequivalence assessment than the 2001 Note for Guidance. It is now recommended that in almost all cases the evaluation of bioequivalence should be based upon the measurement of plasma concentrations of the parent compound. The rationale for this approach is that the concentration-time profile of the parent drug is more sensitive to changes in formulation performance than that of the metabolite, which includes the processes of metabolite formation, distribution and elimination $(23,24)$. The use of metabolite concentrations to assess bioequivalence can only be considered if it is adequately demonstrated that the existing analytical methods for measurement of the parent compound are not sensitive enough to accurately determine the single-dose parent drug plasma concentration-time curve, and cannot be improved by using state-of-the-art techniques. Even for inactive prodrugs, demonstration of bioequivalence based on parent compound plasma concentration is generally recommended.

In the EMA Questions \& Answers document the examples of clopidogrel and losartan are given (10). Clopidogrel, a platelet aggregation inhibitor, is an inactive prodrug undergoing extensive presystemic metabolism to an active metabolite, clopidogrel thiol, and an inactive carboxylic acid metabolite. Due to the chemical instability of clopidogrel thiol and its low plasma concentrations, its quantification in plasma is problematic. Moreover, the plasma concentrations of the parent compound are also extremely low and much lower (up to 2000 fold) than those of the inactive carboxylic acid metabolite $(25,26)$. Consequently, at the time of approval of the reference product (Plavix ${ }^{\circledR}$ ) the pharmacokinetic characteristics of clopidogrel were established based on the pharmacokinetics of the inactive carboxylic acid metabolite. Recently, sensitive liquid chromatography/tandem mass spectrometric methods have been developed for the quantification of the parent compound, clopidogrel, in human plasma which allow the accurate determination of the clopidogrel plasma concentration-time profile following a single 75 mg dose administration of the drug (26-30). As a consequence, the CHMP Pharmacokinetics Working Party of the EMA now requires bioequivalence assessment between reference and generic clopidogrel formulations based on plasma concentrations of the parent prodrug (10). Also for losartan, a selective angiotensin II type 1 receptor antagonist undergoing metabolism to the 5-carboxylic acid derivative, which is 10 - to 40 times more potent than losartan itself, the EMA recommends $\mathrm{BE}$ assessment based on parent compound plasma concentrations (10).

To demonstrate bioequivalence for a generic mycophenolate mofetil product, on the contrary, the EMA exceptionally recommends the use of metabolite data only (10). Mycophenolate mofetil is an inactive ester prodrug of mycophenolic acid (32). Following oral administration, mycophenolate mofetil is rapidly and extensively metabolized by esterases to its active metabolite mycophenolic acid. The parent compound, mycophenolate mofetil, has a half-life of less than 1 hour and maximum plasma concentrations are already reached after approximately $30 \mathrm{~min}$. This short $\mathrm{t}_{\max }$ limits the reliable estimation of $\mathrm{C}_{\max }$ of this prodrug and, consequently, the EMA accepts bioequivalence assessment based on the active metabolite, mycophenolic acid, without measurement of the parent compound itself.

The Office of Generic Drugs of the FDA publishes product-specific bioequivalence recommendations (14). The FDA usually recommends bioequivalence to be based on the calculation of a $90 \%$ confidence interval for the parent compound. It is interesting that for some drug substances, e.g. losartan (but not clopidogrel), the FDA recommends to assess bioequivalence based on the $90 \%$ confidence interval for the parent compound but additionally recommends submission of metabolite data "as supportive evidence of therapeutic outcome" (33). How exactly these metabolite data could be interpreted as supporting, or possibly not supporting the outcome of the BE study based on 
the $90 \%$ confidence interval for AUC and $\mathrm{C}_{\max }$ of the parent compound, is not clarified.

\section{STATISTICAL ANALYSIS AND ACCEPTANCE CRITERIA}

Bioequivalence assessment is based on the "two one-sided tests" procedure in which the $90 \%$ confidence interval (CI) around the geometric mean ratio (GMR) of the test and reference values of an appropriate bioavailability measure, i.e. $\mathrm{AUC}_{0-\mathrm{t}}$ (or $\mathrm{AUC}_{0-72 \mathrm{~h}}$ ) and $\mathrm{C}_{\max }$, is required to fall within preset BE limits, which normally are 80.00 to $125.00 \%$ (34). These BE metrics should be analysed using analysis of variance (ANOVA). The terms to be used in the ANOVA model are usually sequence, subject within sequence, period and formulation. According to the 2010 EMA bioequivalence guideline fixed effects, rather than random effects, should be used for all terms. A non-parametric analysis is not acceptable. A statistical evaluation of $t_{\max }$ is not required, but in the case where rapid release is clinically relevant or is related to adverse events there should be no apparent difference in median $t_{\max }$ and its variability between test and reference products.

\section{HIGHLY VARIABLE DRUGS OR DRUG PRODUCTS}

The 2010 EMA guideline now recommends that bioequivalence for highly variable drugs or drug products (HVDP's) can be assessed by using a wider acceptance range for the $90 \%$ confidence interval of $\mathrm{C}_{\max }$, but not for AUC, compared to the usual $80.00-125.00 \%$ acceptance limits. The widening of the acceptance interval for $\mathrm{C}_{\max }$ should be clinically justified, and the bioequivalence study, which should be of a replicate design, must demonstrate that the within-subject variability for $\mathrm{C}_{\max }$ of the reference product $\left(\mathrm{CV}_{\mathrm{WR}}\right)$ is $>30 \%$. The extent of widening the acceptance range for $\mathrm{C}_{\max }$ is based on the within-subject variability of the reference product, observed in the bioequivalence study, using the scaled average bioequivalence (SABE) approach. SABE has been extensively discussed in the scientific literature (35-38).

Highly variable drugs (HVDs) or drug products (HVDPs) are generally defined as those medicinal products showing a high within-subject variability, i.e. $\mathrm{CV}_{\mathrm{w}}>30 \%$, of the $\mathrm{BE}$ metrics AUC and/or $\mathrm{C}_{\max }(39,40)$. Bioequivalence parameter variability can be due to characteristics of the drug substance itself, e.g. extensive presystemic metabolism, or due to drug product formulation variability. Usually $\mathrm{C}_{\max }$ shows a higher within-subject variability than AUC. Of 212 bioequivalence studies submitted to the FDA between 2003 and 2005, 33 studies showed a high within-subject variability in AUC and/or $C_{\max }$ (40). In 28 of the 33 studies, only $\mathrm{C}_{\max }$, but not AUC, showed a $\mathrm{CV}_{\mathrm{w}}>30 \%$. Not one study showed high variability for AUC when variability of $\mathrm{C}_{\max }$ was moderate or low (i.e. $\mathrm{CV}_{\mathrm{WR}}<30 \%$ ). When the within-subject variability in AUC and/or $\mathrm{C}_{\max }$ is high the estimated $90 \% \mathrm{CI}$ is very wide and will exceed the usual $80.00-125.00 \%$ acceptance limits unless a large number of study subjects are included in the BE study. This may lead to situations where bioequivalence cannot be established even when the reference formulation is tested against itself $(41,42)$. At the 1995 AAPS/FDA Workshop on the Evaluation of Orally Administered Highly Variable Drugs and Drug Formulations, the approach of widening the acceptance limits for highly variable drugs/drug products was put forth as a major recommendation to the FDA (43). In the EMA Note for Guidance on the Investigation of Bioavailability and Bioequivalence of 2001, widening the usual acceptance limits of 80.00 $125.00 \%$ to $75.00-133.00 \%$ was allowed in certain cases for $\mathrm{C}_{\max }$, and in rare cases also for AUC, provided there were no safety or efficacy concerns with the relevant drug substance. Instead of widening the bioequivalence acceptance limits from $80.00-125.00 \%$ to $75.00-133.00 \%$ for all drugs/drug products showing a $\mathrm{CV}_{\mathrm{w}}>30 \%$, a scientifically more appealing approach is to widen the acceptance limits based on the within-subject variability of the reference formulation $\mathrm{CV}_{\mathrm{WR}}$, i.e. the so-called scaled average bioequivalence (SABE) method (35-38). The recommendation of using reference-scaling is based on the general concept that reference variability should be used as an index for setting the public standard expressed in the BE acceptance limit.

For drugs/drug products with low to moderate within-subject variability bioequivalence is usually declared if the difference between the logarithmic means of $\mathrm{AUC}$ or $\mathrm{C}_{\max }$ for test and reference product ( $\mu_{\mathrm{T}}$ and $\mu_{\mathrm{R}}$, respectively) lies between preset bioequivalence limits $\theta_{\mathrm{A}}$. Therefore, average bioequivalence (ABE) is accepted if the following criterion is satisfied:

$$
-\theta_{\mathrm{A}} \leq \mu_{\mathrm{T}}-\mu_{R} \leq+\theta_{A}
$$

The limits $\theta_{\mathrm{A}}$ are generally symmetrical on the logarithmic scale and usually equal to $\ln (1.25)$. 
True values of the population means $\mu_{\mathrm{T}}$ and $\mu_{\mathrm{R}}$ are not known and, therefore, their estimates, i.e. $\mathrm{m}_{\mathrm{T}}$ and $\mathrm{m}_{\mathrm{R}}$, have to be used:

$$
-0.223 \leq \mathrm{m}_{\mathrm{T}}-\mathrm{m}_{\mathrm{R}} \leq+0.223 \quad \text { [eq. 2] }
$$

For HVDs/HVDPs when the within-subject variability of the reference product exceeds a preset "switching" value $\left(\mathrm{CV}_{\mathrm{WR}}\right.$ of $30 \%$ corresponding to a "switching" standard deviation $\sigma_{\mathrm{s}}$ of 0.294$)$, the difference between logarithmic means, i.e. $\mu_{\mathrm{T}}-\mu_{\mathrm{R}}$, can be normalized (scaled) to the within-subject variability of the reference formulation as follows:

$$
\begin{aligned}
& -\frac{0.223}{\sigma_{0}} \leq \frac{m_{\mathrm{T}}-m_{\mathrm{R}}}{\mathrm{s}_{\mathrm{WR}}} \leq+\frac{0.223}{\sigma_{0}} \\
& \text { or: } \\
& -\left(\frac{0.223}{\sigma_{0}}\right) \cdot \mathrm{s}_{\mathrm{WR}} \leq m_{\mathrm{T}}-m_{\mathrm{R}} \leq+\left(\frac{0.223}{\sigma_{0}}\right) \cdot \mathrm{s}_{\mathrm{WR}} \text { [eq. 3] }
\end{aligned}
$$

where $\sigma_{0}$ is the "regulatory standard deviation" which can be set by the regulatory authorities, and $\mathrm{S}_{\mathrm{WR}}$ represents the within-subject standard deviation of the reference formulation (both on the logarithmic scale). This means that the usual bioequivalence limits $(-0.223$ and +0.223$)$ are expanded in proportion to the within-subject variability of the reference formulation $\mathrm{s}_{\mathrm{WR}}$, starting from the "switching" variability, as follows:

$$
\left(\frac{0.223}{\sigma_{0}}\right) \cdot \mathrm{s}_{\mathrm{WR}}=\mathrm{k} \cdot \mathrm{s}_{\mathrm{WR}} \quad \text { [eq. 5] }
$$

where $0.223 / \sigma_{0}$ is the proportionality or regulatory constant $\mathrm{k}$. As mentioned above, HVDs/HVDPs are defined to have a withinsubject coefficient of variation exceeding $30 \%$, i.e. $\mathrm{CV}_{\mathrm{WR}}>30 \%$. Because the $\mathrm{CV}_{\mathrm{WR}}$ is related to the standard deviation on the logarithmic scale $\mathrm{s}_{\mathrm{WR}}$ as follows:

$$
\mathrm{CV}_{\mathrm{WR}}=\left[\exp \left(\mathrm{s}_{\mathrm{WR}}^{2}\right)-1\right]^{1 / 2} \quad \text { [eq. 6] }
$$

a $\mathrm{CV}_{\mathrm{WR}}$ of $30 \%$ corresponds to a "switching" variability $\sigma_{\mathrm{s}}=0.294$. The EMA has chosen a regulatory standard deviation $\sigma_{0}$ equal to the switching variability $\sigma_{\mathrm{s}}$, i.e. 0.294 , and therefore the regulatory constant $\mathrm{k}$ is equal to 0.760 . This means that the acceptance limits for HVDs/HVDPs using the average bioequivalence approach with expanding limits (ABEL), a variant of the scaled average bioequivalence (SABE) method, are:

$$
[\mathrm{U}, \mathrm{L}]=e^{\left( \pm 0.76 \cdot \mathrm{s}_{\mathrm{WR}}\right)}
$$

Widening of the usual $\mathrm{BE}$ acceptance limits of $0.80-1.25$ according to this method recommended by the EMA is only acceptable for $\mathrm{C}_{\max }$. Provided there are no safety/efficacy concerns, the BE acceptance limits can be widened to a maximum of $69.84-143.19 \%$, corresponding to a $\mathrm{CV}_{\mathrm{WR}}$ of $50 \%$ (Fig. 1). An additional constraint imposed by the EMA is that the GMR for $\mathrm{C}_{\max }$ should lie within the conventional $80.00-125.00 \%$ acceptance range.

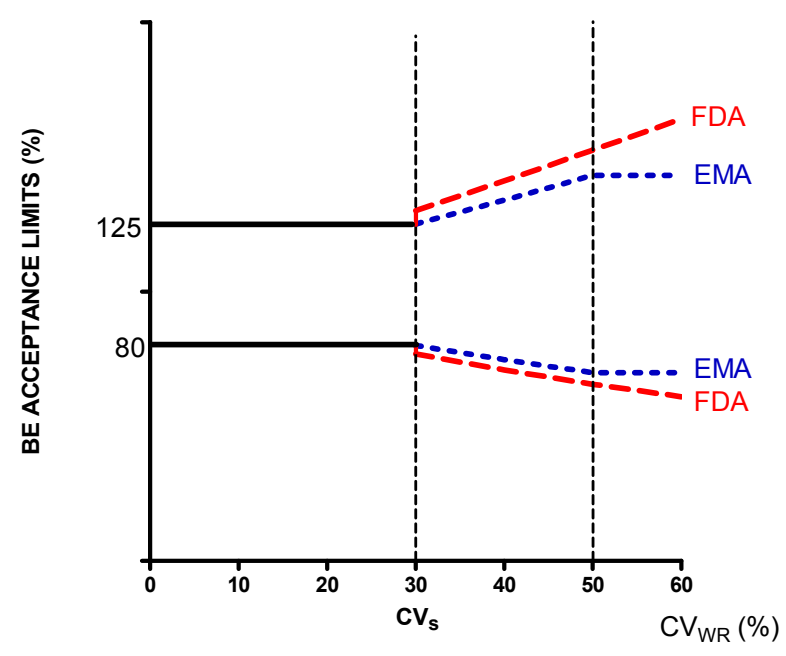

Figure 1. The EMA regulatory limits for bioequivalence acceptance are $80.00-125.00$ until a within-subject switching coefficient of variation $\left(\mathrm{CV}_{\mathrm{s}}\right)$ of $30 \%$ is reached. For $\mathrm{CV}_{\mathrm{WR}}$ values between 30 and $50 \%$, the bioequivalence acceptance limits are expanded according to the within-subject variability of the reference formulation (ABEL approach). For $\mathrm{CV}_{\mathrm{WR}}$ values of $50 \%$ or higher the $90 \% \mathrm{BE}$ acceptance limits are capped at $69.84-143.19 \%$. The FDA recommends scaling of the usual bioequivalence limits $(80-125 \%)$ starting at a $\mathrm{CV}_{\mathrm{WR}}$ of $30 \%$. However, the EMA and FDA use a different regulatory standardized variability $\left(\sigma_{0}\right)$, i.e. 0.294 and 0.25 , respectively. This difference in the choice of $\sigma_{0}$ explains why the FDA expanded limits (red lines) are not only discontinuous at the switching variability but also wider than the EMA expanded limits (blue lines).

The use of the ABEL approach necessitates a replicate study design allowing precise estimation of the within-subject variability of $\mathrm{C}_{\max }$ for the reference formulation. As stated in the 2010 EMA 
Guidance on the Investigation of Bioequivalence, either a 3-period or a 4-period replicate crossover design is acceptable. Haidar et al. (2008) proposed a 3-period, reference-replicated, crossover design with sequences of TRR, RTR and RRT (37). They favoured the 3-period design over the 4-period design where both test $(\mathrm{T})$ and reference $(\mathrm{R})$ are administered twice because the subject withdrawal rate increases with the length of the study. The only advantage of the 4-period design is that it allows the calculation of the variability of the test formulation, but this variability is not used in the proposed statistical method. The EMA Questions \& Answers document contains a section "Clarification on the recommended statistical method for the analysis of a bioequivalence study" in which the statistical analysis of data from a 3-period ( $\mathrm{R}$ administered twice, $\mathrm{T}$ administered once) and a 4-period ( $\mathrm{R}$ and $\mathrm{T}$ administered twice) crossover study are shown in detail (10).

The FDA now also recommends the reference-scaled average bioequivalence approach when the BE parameters AUC and/or $\mathrm{C}_{\max }$ show high within-subject variability, i.e. $\geq 30 \% \mathrm{CV}_{\mathrm{WR}}$ $[37,44]$. However, the FDA uses a regulatory standard variability, $\sigma_{0}$, of 0.25 . As a result, since scaling starts at a $\mathrm{CV}_{\mathrm{WR}}$ of $30 \%$, the $\mathrm{BE}$ limits are discontinuous at the switching variability (Fig. 1). This is different from the ABEL procedure proposed by the EMA where scaling also starts at a "switching" variability of $30.0 \%$ but because the EMA regulatory standard variability $\sigma_{0}$ is set at 0.294, the BE limits are continuous (Fig. 1) and more conservative. In addition, unlike the EMA which only allows scaling for $\mathrm{C}_{\max }$, the FDA allows scaling for $\mathrm{C}_{\max }$ and $\mathrm{AUC}(45,46)$. Both the EMA and the FDA apply a GMR constraint, i.e. the point estimate for the $T / R$ geometric mean ratio must fall between 0.80 and 1.25 .

\section{NARROW THERAPEUTIC INDEX DRUGS}

The debate as to whether or not it is necessary to apply stricter guidelines for certain drug substances has been on-going for several decades $(47,48)$. According to the revised EMA Guideline on the Investigation of Bioequivalence, for narrow therapeutic index drugs (NTIDs) the usual acceptance interval for $\mathrm{AUC}$, and also for $\mathrm{C}_{\max }$ if necessary for safety, efficacy or drug level monitoring reasons, may need to be tightened to $90.00-111.11 \%$. However, according to the same guideline: "It is not possible to define a set of criteria to categorise drugs as narrow therapeutic index drugs (NTIDs) and it must be decided case by case if an active substance is an NTID based on clinical considerations".

For two drug substances, i.e. cyclosporine and tacrolimus, the EMA has now clearly stated that they should be considered as having a narrow therapeutic index (10). Consequently, based on efficacy and safety considerations, the 90.00$111.11 \%$ acceptance limits are required for cyclosporine for both $\mathrm{AUC}$ and $\mathrm{C}_{\max }$. For tacrolimus, on the other hand, the acceptance criterion is only tightened to $90.00-111.11 \%$ for AUC but nor for $\mathrm{C}_{\max }$ where the usual 80.00$125.00 \%$ acceptance limits apply (10).

There is no consensus among the various EU member states on the issue of bioequivalence acceptance criteria as well as switchability between innovator and generic medicinal products. For example, the Danish Health and Medicines Authority considers that the $90 \%$ confidence interval for the ratio test versus reference (GMR) of both AUC and $\mathrm{C}_{\max }$ should incorporate $100 \%$ irrespective of whether acceptance limits of $80-125 \%$ or narrower are employed. Deviations may be accepted if they can be adequately justified not to have impact on either the overall therapeutic effect or safety profile of the product (49). This requirement is not part of the EMA recommendations. Like the EMA, however, the Danish Health and Medicines Authority also requires tighter acceptance limits, i.e. $90.00-111.11 \%$, for both $\mathrm{AUC}$ and $\mathrm{C}_{\max }$ for substances with a narrow therapeutic index with regard to automatic substitution for the following substances or therapeutic classes: aminophylline/theophylline, lithium, vitamin $\mathrm{K}$ antagonists, antiepileptics apart from levetiracetam and benzodiazepines, antiarrhythmics, centrally acting anorectics and tricyclic antidepressants (49). The Danish authorities clearly accept the idea that medicinal products containing the NTIDs on their list are therapeutically equivalent because stricter bioequivalence criteria were applied, and they are therefore considered switchable and, consequently, generic substitution of NTIDs is authorised except for thyroxine and the immunosuppressants cyclosporine and tacrolimus (50).

The Federal Agency for Medicines and Health Products (FAMHP) of Belgium published a list with 31 drug substances considered to have a narrow therapeutic index or to be highly toxic (51). First of all, although there is a substantial overlap between the substances on the Belgian and Danish lists, both lists are certainly not identical. Secondly, medicinal products 
containing one of these 31 substances on the Belgian list are considered to be "non-switchable" and switching from the innovator to the generic medicine, or vice versa, after initiation of therapy is, therefore, discouraged by the Belgian agency. This means that the Belgian authorities, unlike the Danish Health and Medicines Authority, do not accept therapeutic equivalence, and therefore switchability, between medicinal products containing an active substance with a narrow therapeutic index, even though bioequivalence has been demonstrated and for some of them even based on the stricter acceptance limits of 90.00 to $111.11 \%$. This example illustrates how the health authorities of two EU member states interpret and apply the bioequivalence guidelines quite differently. Moreover, with the exception of cyclosporine and tacrolimus, it is currently not known to the health practitioners for which generic medicines, authorized in member states of the EU, bioequivalence with the innovator was demonstrated on the basis of the stricter 90.00$111.11 \%$ acceptance range.

Whether tighter acceptance criteria should be applied to certain drug substances is a controversial issue which has received much attention in the scientific literature and has led to different recommendations by various health authorities (see e.g. 52-56). The Health Protection and Food Branch (HPFB) of Canada issued a specific Guidance for Industry on the bioequivalence requirements for critical dose drugs (57). According to this guidance "critical dose drugs" are defined as those drugs for which comparatively small differences in dose or concentration lead to dose- and concentrationdependent, serious therapeutic failures and/or serious adverse drug reactions. For these "critical dose drugs" the $90 \%$ CI of the relative mean AUC of the test to reference formulation should lie within 90-112\%, according to Canada's HPFB guidance. In addition, the $90 \%$ CI of the relative mean $\mathrm{C}_{\max }$ of the test to reference formulation for these "critical dose drugs" should be between 80 to $125 \%$. For "uncomplicated" drugs, Canada's HPFB does not require calculation of the $90 \% \mathrm{CI}$ for $\mathrm{C}_{\max }$ but requires that the GMR should lie between 80 and $125 \%$ (16). These requirements for "critical dose drugs" are to be met in both the fasted and fed states. In an appendix to the HPFB guidance a list of 9 "critical dose drugs" is given: cyclosporine, digoxin, flecainide, lithium, phenytoin, sirolimus, tacrolimus, theophylline and warfarin (57). The FDA Guidance for Industry on Bioavailability and Bioequivalence Studies for Orally Administered Drug Products recommends that the usual BE limit of 80 to $125 \%$ for nonnarrow therapeutic index drugs remain unchanged for the bioavailability measures ( $\mathrm{AUC}$ and $\mathrm{C}_{\max }$ ) for NTI drug substances unless otherwise indicated by a specific guidance (58). The FDA has long supported the view that stricter acceptance limits for NTIDs are not necessary for purposes of therapeutic substitution (59). However, recently the FDA has re-started to debate the issue whether the current bioequivalence criteria used to approve generic drugs are appropriate for all drugs and specifically whether NTIDs require special considerations. During a recent (July 26, 2011) meeting of the FDA Advisory Committee for Pharmaceutical Science and Clinical Pharmacology, the following $\mathrm{BE}$ assessment approaches for NTIDs were proposed:

1) the use of a replicate study design to quantify the variability of both the reference and the test product, and

2) the use of a scaling approach for $\mathrm{BE}$ assessment $(60,61)$.

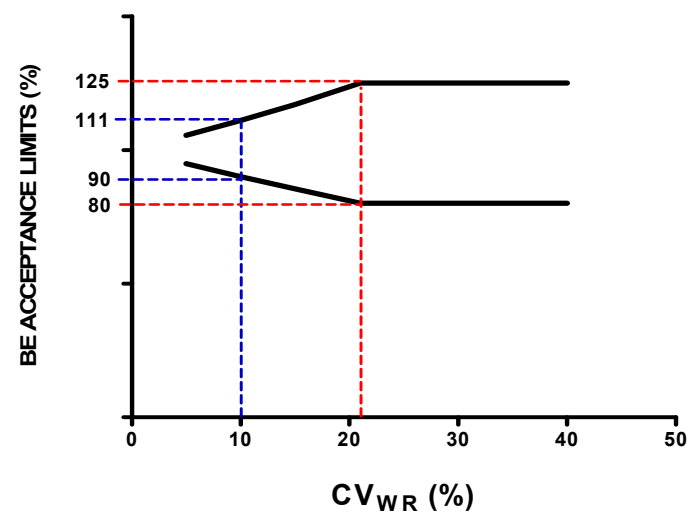

Figure 2. The proposed FDA regulatory default limits for bioequivalence acceptance of NTIDs are 90-111\%. A switching variability $\sigma_{0}$ equal to 0.1 has been proposed by the FDA. This means that at a reference within-subject variability $\mathrm{CV}_{\mathrm{WR}}$ of $10 \%$, the $\mathrm{BE}$ limits are $90-111 \%$. When $\mathrm{CV}_{\mathrm{WR}}$ is greater than $10 \%$, the $\mathrm{BE}$ limits would expand as a function of $\mathrm{CV}_{\mathrm{WR}}$ but the expansion would be capped at $80-125 \%$. This maximum expansion is reached when the $\mathrm{CV}_{\mathrm{WR}}$ is approximately $21 \%$. When the reference variability is less than $10 \%$, the acceptance BE limits would become narrower than the default limits of $90-111 \%$. This approach is similar to the EMA scaled average BE approach for HVDs/HVDPs where the switching variability $\sigma_{0}$ is set by the EMA at 0.294 to give $\mathrm{BE}$ acceptance limits of $80-125 \%$ at a $\mathrm{CV}_{\mathrm{WR}}$ of $30 \%$.

Most NTIDs have a relatively small withinsubject variability, ranging from approximately 5 
to $25 \%(52,62)$. Similar to the scaling approach recommended by the EMA for HVDs/HVDPs, the BE limits would change as a function of the within-subject variability of the reference product $\mathrm{CV}_{\mathrm{WR}}$. The FDA proposes for NTIDs that the default BE limits be $90-111 \%$ and that they be scaled using a switching variability $\left(\sigma_{0}\right)$ of 0.1 (which corresponds to a $\mathrm{CV}_{\mathrm{WR}}$ of $10.03 \%$ ) to a maximum of $80-125 \%$. This switching variability is chosen such that the $\mathrm{BE}$ acceptance limits are $90-111 \%$ when the $\mathrm{CV}_{\mathrm{WR}}$ is equal to $10 \%$. This means that when the $C V_{\mathrm{WR}}$ is less than $10 \%$, the BE limits would narrow beyond the default limits of $90-111 \%$ as a function of the within-subject variability of the reference product. The maximum BE acceptance limits, i.e. 80-125\%, will be reached at a $\mathrm{CV}_{\mathrm{WR}}$ of approximately $21 \%$ (Fig. 2). The FDA is also investigating the impact of additional $\mathrm{BE}$ acceptance criteria for NTIDs such as point estimate limits for $\mathrm{C}_{\max }$ and AUC, and a requirement that the $90 \%$ confidence interval around the GMR for $\mathrm{C}_{\max }$ and $\mathrm{AUC}$ includes $100 \%(61)$.

\section{DOSAGE STRENGTH(S) TO BE INVESTIGATED}

When marketing authorisation is requested for several strengths of a product it may be sufficient to carry out an in vivo bioequivalence study on one or two strengths only and to apply for a biowaiver for the remaining strengths. The choice of the strength(s) at which the in vivo bioequivalence study should be carried out, as well as the conditions that have to be fulfilled to qualify for a biowaiver for additional strengths are explained in much more detail in the 2010 EMA bioequivalence guideline. In this regard it is noteworthy that the pharmacokinetics are considered to be linear (or dose proportional) if the difference in dose-adjusted AUCs is not more than $25 \%$ between the concerned strengths, i.e. the strength for which an in vivo bioequivalence study is carried out and the one for which a biowaiver is requested. The in vivo bioequivalence study should generally be conducted at the highest strength. Selection of a lower strength is acceptable for products with dose proportional pharmacokinetics and where the drug substance is highly soluble according to the BCS. Other considerations taken into account to select the strength at which to carry out the BE study are tolerability/safety of the study subjects and insufficient sensitivity of the bioanalytical method to determine the plasma concentrationtime profile. One of the conditions that have to be fulfilled to extrapolate the results of a BE study carried out with one of the strengths only is related to the composition of the various strengths which should be proportional, i.e. having a constant ratio between the active substance and the various excipients (some minor deviations of the rule are accepted). When a BE study has to be performed on more than two strengths because the condition of proportional composition is not fulfilled, the bracketing approach may be used, i.e. the strengths selected for the BE studies represent the extremes in strength or in composition.

\section{BCS-BASED BIOWAIVERS}

The 2001 EMA Note for Guidance on the Investigation of Bioavailability and Bioequivalence referred to exemptions from $\mathrm{BE}$ studies based on the concepts underlying the BCS (5). However, the description of the criteria on which a biowaiver may be granted in this 2001 EMEA Note for Guidance was much less detailed (less than one page!) than in the FDA Guidance for Industry on BCS-based biowaivers. In 2007 a Concept Paper was published by the EMA in which it was noted that BCS-based biowaivers were rarely used in the EU probably due to uncertainties by the sponsors as well as the regulators on how to interpret these EMEA guidelines (7). This 2007 EMA Concept Paper announced the preparation of a document which would address the issue of BCS-based biowaivers in more detail to reach an optimal and harmonised application of biowaiver principles. The EMA BCS-based biowaiver guidelines are now described in Appendix III of the revised 2010 Guideline for the Investigation of Bioequivalence (9).

Unlike the FDA which only accepts biowaivers for BCS class I substances (high solubility, high permeability), the EMA now considers biowaivers for BCS class I and III substances (high solubility, low permeability). It has been argued in several scientific commentaries that BCS class III substances would be good candidates for biowaivers (63-67). A drug substance is considered highly soluble, according to the EMA guideline, "if the highest single dose administered as immediate release formulation(s) is completely dissolved in $250 \mathrm{ml}$ of buffers within the range of $\mathrm{pH} 1.0-6.8$ at $37 \pm$ $1{ }^{\circ} \mathrm{C}$ " (9). The 2010 EMA guideline defines permeability as a function of extent of absorption following oral administration: "Complete absorption is generally related to high 
permeability". When the measured extent of absorption, based on human data (absolute bioavailability, mass-balance studies), is at least $85 \%$, complete absorption is accepted. Although the EMA accepts in vitro permeability investigations as supportive evidence of in vivo human data, the FDA seems to attach greater importance to in vitro studies (cell cultures, intestinal tissue) and in vivo/in situ perfusion experiments in animal models. In the case of biowaivers for class III substances the in vitro dissolution of the test and reference product has to be very rapid ( $>85 \%$ within $15 \mathrm{~min})$, and special attention must be paid to the excipients since it is known that the absorption of BCS class III substances is more susceptible to transportermediated excipient-drug interactions (69). For BCS class I substances the EMA advises the use of similar amounts of the same excipients in the test product compared to the reference product. For BCS class III substances, according to the EMA the excipients have to be qualitatively the same and quantitatively very similar in test and reference preparation.

The World Health Organization (WHO), which is not a regulatory body but publishes technical reports and guidelines which are recommendations to national authorities, not only allows biowaivers for BCS class I and BCS class III substances but also under certain circumstances for class II substances $(18,70)$. This lack of harmonisation creates confusion which in turn leads to suspicion by health care providers and patients, especially since many national authorities of third world countries give these WHO reports regulatory status. All stakeholders in the development and registration of new drug products must balance the need for scientific rigor in assuring $\mathrm{BA} / \mathrm{BE}$ (and hence product quality towards consistent therapeutic outcomes) with the time and expense of conducting in vivo $\mathrm{BE}$ studies, and the overall impact on product costs and timely availability to patients. Ideally these guidelines should be the same worldwide to ensure that patients all over the world can benefit from affordable and safe medicinal products.

\section{GLOBAL HARMONIZATION?}

The generic drug approval process has evolved over the past 35 years and regulatory agencies in a number of western countries have now established stringent requirements for the design, performance and evaluation of BE studies to protect the consumer of being exposed to drug products of inferior quality. Although the current
$\mathrm{BE}$ guidelines and recommendations of the major regional and national health authorities show a fair degree of consistency, a number of outstanding $\mathrm{BE}$ issues and concerns remain to be resolved. The most obvious of these controversial issues, such as the BE acceptance limits for NTI drugs and HVDs/HVDPs, the use of metabolites for $\mathrm{BE}$ assessment, conditions to grant biowaivers, are not always dealt with in the same way by the various health authorities. Global harmonization should therefore be the next logical step in the continuing process to improve the $\mathrm{BE}$ guidelines as a means to guarantee safe and efficacious drug products for the consumer in all parts of the world. Global harmonization efforts by the International Conference on Harmonization (ICH) and the WHO should be stepped up in collaboration with the regulatory agencies of the western world as more nations throughout the world have come to rely on lowcost, good-quality multi-source (generic) pharmaceutical products to provide lower healthcare costs without sacrificing important public health goals. However, as pointed out above, consensus on a number of $\mathrm{BE}$ issues have not been reached at this point in time amongst international regulatory agencies. In addition, differing levels of commitment and resources by the various countries and regions constitute another formidable barrier that has to be overcome to harmonize $\mathrm{BE}$ approaches to ensure development of optimally performing and affordable drug products for use by health practitioners and patients in the global community.

\section{CONCLUSION}

Compared to the 2001 EMA Note for Guidance on the Investigation of Bioavailability and Bioequivalence (5), the 2010 revised guidelines give clearer recommendations regarding such topics as the assessment of $\mathrm{BE}$ for HVD's/HVDP's, the very limited use of metabolites in BE assessment, the dose strength to be used in case of application for marketing authorization of several strengths, and BCS-based and other biowaivers. Unfortunately, global harmonization was certainly not an objective of these revised EMA guidelines.

\section{REFERENCES}

1. Nation RL, Sansom LN. Bioequivalence requirements for generic products. Pharmac Ther 1994; 62: 41-55. 
2. Midha KK, McKay G. Bioequivalence: its history, practice and future. AAPS J 2009; 11: 664-70.

3. CPMP Note for Guidance on the Investigation of Bioavailability and Bioequivalence, Brussels, December 1991.

4. Pharmacokinetic studies in man, 1987 (http://www.emea.europa.eu/pdfs/human/ewp/3cc 3aen.pdf).

5. EMEA Note for Guidance on the Investigation of Bioavailability and Bioequivalence, London, July 26, 2001, $\quad$ CPMP/EWP/QWP/EMEA (http:/www.emea.europa.eu/pdfs/human/qwp/140 198enfin.pdf).

6. EMEA Recommendation on the Need for Revision of CHMP "Note for Guidance on the Investigation of Bioavailability and Bioequivalence", London, May 2007, EMEA/CHMP/EWP/200943/2007.

7. EMEA Concept Paper on BCS-Biowaiver, EMEA/CHMP/EWP/213035/2007, May 20, 2007 (http://www.emea.europa.eu/pdfs/human/ewp/213 03507en.pdf).

8. Verbeeck RK, Warlin J. The European Union, in Kanfer I, Shargel L (eds), Generic Drug Product Development: International Regulatory Requirements for Bioequivalence, Informa Healthcare, New York, NY, pp. 95-113, 2010.

9. EMEA Guideline on the Investigation of Bioequivalence, CPMP/EWP/QWP/1401/98 Rev. 1/Corr **, London, January 20, 2010.

10. EMA Questions \& Answers: Positions on specific questions addressed to the Pharmacokinetics Working Party, EMA/618604/2008 Rev. 3, February 16, 2012.

11. EMEA Guideline on Clinical Development of Fixed Combination Medicinal Products, CHMP/EWP/240/95 Rev. 1, February 19, 2009 http:/www.ema.europa.eu/docs/en_GB/document _library/Scientific_guideline/2009/09/WC500003 686.pdf

12. EMEA Note for Guidance on Modified Release Oral and Transdermal Dosage Forms: Section II (Pharmacokinetic and Clinical Evaluation), CPMP/EWP/280/96Corr * London, July 28,1999, (http://www.ema.europa.eu/docs/en_GB/documen t_library/Scientific_guideline/2009/09/WC500003 126.pdf

13. EMEA Concept Paper on the need for revision of the note for guidance on modified release oral and transdermal dosage forms: section II (pharmacokinetic and clinical evaluation), EMA/CHMP/EWP/1303/2010,May 20, 2010 (http://www.ema.europa.eu/docs/en_GB/documen t library/Scientific guideline/2010/06/WC500091 662.pdf).

14. FDA Guidance for Industry: Bioequivalence Recommendations for Specific Products, CDER/FDA, Washington, June 2010 (http://www.fda.gov/downloads/Drugs/GuidanceC omplianceRegulatoryInformation/Guidances/ucm0 72872.pdf).
15. Hauschke D, Steinijans VW, Diletti E, Burke M. Sample size determination for bioequivalence assessment using a multiplicative model. $J$ Pharmacokinet Biopharm 1992; 20: 557-61.

16. HPFB Guidance for Industry: Conduct and Analysis of Bioavailability and Bioequivalence Studies - Part A: Oral Dosage Formulations, not in Modified used for Systemic Effects, Ottawa, $1992 \quad$ (http://www.hc-sc.gc.ca/dhpmps/alt_formats/hpfb-dgpsa/pdf/prodpharma/bioa-eng.pdf).

17. NIHS Japan Guideline for Bioequivalence Studies of Generic Products, December 2006 http://www.nihs.go.jp/drug/be-guide(e)/be2006e .pdf

18. WHO Expert Committee on Specifications for Pharmaceutical Preparations, Annex 7: Multisource (generic) pharmaceutical products guidelines on registration requirements to establish interchangeability. WHO Technical Report Series No. 937, 2006 (http://www.who.int/medicines/publications/phar mprep/PDF_TRS953_WEB.pdf).

19. Pocock SJ. Group sequential methods in the design and analysis of clinical trials.Biometrika 1977; 64: 191-99.

20. Potvin D, DiLiberti CE, Hauck WW, Parr AF, Schuirmann DJ, Smith RA. Sequential design approaches for bioequivalence studies with crossover designs. Pharmaceut Stat 2008; 7: 245 62.

21. Montague TH, Potvin D, DiLiberti CE, Hauck WW, Parr AF, Schuirmann DJ. Additional results for "Sequential design approaches for bioequivalence studies with crossover designs. Pharmaceut. Stat. 10, published on-line Feb 10, 2011.

22. Common Technical Document for the Registration of Pharmaceuticals for Human Use: Organisation of Common Technical Document, CPMP/ICH/2887/99, London, February 2004.

23. Jackson AJ, Robbie G, Marroum P. Metabolites and bioequivalence: past en present. Clin Pharmacokinet 2004; 43: 655-72.

24. Midha KK, Rawson MJ, Hubbard JW. The role of metabolites in bioequivalence. Pharm Res 2004; 21: 1331-44.

25. Plosker GL, Lyseng-Williamson KA. Clopidogrel - a review of its use in the revention of thrombosis. Drugs 2007; 67: 613-46.

26. Lenz TL, Wilson AF. Clinical pharmacokinetics of antiplatelet agents used in the secondary prevention of stroke. Clin Pharmacokinet 2003; 42: 909-20.

27. Nirogi RV, Kandikere VN, Shukla M, Mudigonda K, Maurya S, Boosi R. Quantification of clopidogrel in human plasma by sensitive liquid chromatography/tandem mass spectrometry. Rapid Commun Mass Spectrom 20: 1695-1700.

28. Silvestro L, Gheorghe MC, Tarcomnicu J, Savu S, Savu SR, Iordachescu A, Dulea C.Development 
and validation of an HPLC-MS/MS method to determine clopidogrel in human plasma: use of incurred samples to test back-conversion. $J$ Chromatogr B 2010; 878: 3134-42.

29. Richter W, Erenmemisoglu A, Van der Meer MJ, Emritte N, Tuncay E, Koytchev R. Bioequivalence study of two different clopidogrel bisulfite filmcoated tablets. Arzneim-Forsch/Drug Res 2009; 59: 297-302.

30. El Ahmady O, Ibrahim M, Hussain AM, Bustami RT. Bioequivalence of two oral formulations of clopidogrel tablets in healthy male volunteers. Int J Clin Pharmacol Ther 2009; 47: 780-84.

31. Di Girolamo G, Czerniuk P, Bertuola R, Keller GA. Bioequivalence of two tablet formulations of clopidogrel in healthy Argentinian volunteers: a single-dose, randomized-sequence, open-label crossover study. Clin Ther 2010; 32: 161-70.

32. Staatz CE, Tett SE. Clinical pharmacokinetics and pharmacodynamics of mycophenolate in solid organ transplant recipients. Clin Pharmacokinet 2007; 46:13-58.

33. Guidance on Losartan Potassium, FDA, May 2008 (http://www.fda.gov/Downloads/Drugs/Guidance ComplianceRegulatoryInformation/Guidances/uc m088645.pdf).

34. Schuirmann DJ. A comparison of the two onesided tests procedure and the power approach for assessing the equivalence of average bioavailability. J Pharmacokinet Biopharm 1987; 15: 657-80.

35. Boddy WO, Snikeris FC, Kringle RO, Wei GCG, Oppermann JA, Midha KK. An approach for widening the bioequivalence acceptance limits in the case of highly variable drugs. Pharm Res 1995; 12: 1865-68.

36. Tothfalusi L, Endrenyi L, Midha KK, Rawson MJ, Hubbard JW. Evaluation of bioequivalence of highly-variable drugs and drug products. Pharm Res 2001; 18:728-33.

37. Haidar SH, Davit B, Chen M-L, Conner D, Lee LM, Li QH, Lionberger R, Makhlouf F, Patel D, Schuirmann DJ, Yu LX. Bioequivalence approaches for highly variable drugs and drug products. Pharm Res 2008; 25: 237-41.

38. Tothfalusi L, Endrenyi L, Garcia Arieta A. Evaluation of bioequivalence for highly variable drugs with scaled average bioequivalence. Clin Pharmacokinet 2009; 48: 725-43.

39. Blume H, Midha KK. Bio-international '92, conference on bioavailability, bioequivalence and pharmacokinetic studies: Bad Homburg, Germany, 20-22, May 1992. Eur J Pharm Sci 1993; 1: 165 71.

40. Davit BM, Conner DP, Fabian-Fritsch B, Haidar S, Jiang X, Patel DT, Seo PRH, Suh K, Thompson CL, Yu LX. Highly variable drugs: observations from bioequivalence data submitted to the FDA for new generic drug applications. AAPS J 2008; 10: $148-56$.
41. Midha KK, Rawson MJ, Hubbard JW. The bioequivalence of highly variable drugs and drug products. Int J Clin Pharmacol Ther 2005; 43: 485-98.

42. Tsang YC, Pop R, Gordon P, Hems J, Spino M. High variability in drug pharmacokinetics complicates determination of bioequivalence: experience with verapamil. Pharm Res 1996; 13: 846-50.

43. Shah VP, Yacobi A, Barr WH, Benet LZ, Breimer D, Dobrinska MR, Endrenyi L, Fairweather W, Gillespie W, Gonzales MA, Hooper J, Jackson A, Lesko LJ, Midha KK, Noonan PK, Pataik R, Williams RL. Evaluation of orally administered highly variable drugs and drug formulations. Pharm.Res 1996; 13:1590-94.

44. FDA Draft Guidance on Progesterone, February 2011(http://www.fda.gov/downloads/drugs/Guida nceComplianceRegulatoryInformation/Guidances/ UCM209294.pdf).

45. Tothfalusi L, Endrenyi L. Sample sizes for designing bioequivalence studies for highly variable drugs. J Pharm Pharmaceut Sci 2012; 15: $73-84$

46. Karalis V, Symillides M, Macheras P. Bioequivalence of highly variable drugs: a comparison of the newly proposed regulatory approaches by FDA and EMA. Pharm Res 2012; 29: 1066-77.

47. Benet LZ, Goyan JE. Bioequivalence and narrow therapeutic index drugs. Pharmacotherapy 1995; 15: 433-40.

48. Levy G. What are narrow therapeutic index drugs? Clin Pharmacol Ther 1998; 63: 501-05.

49. Danish Health and Medicines Authority: Bioequivalence and labelling of medicinal Products with regard to generic substitution, February $\quad 07, \quad 2012$ (http://laegemiddelstyrelsen.dk/en/topics/authorisa tion-and-supervision/licensing-of-

medicines/marketing-authorisation/applicationfor-marketing-authorisation/bioequivalence-andlabelling-of-medicine--bstitution).

50. Danish Health and Medicines Authority: Generic substitution terminated for oral medicines containing cyclosporine or tacrolimus, July 13, 2011(http://laegemiddelstyrelsen.dk/en/topics/auth orisation-and-supervision/licensing-ofmedicines/news/generic-substitution-terminatedfor-oral--tacrolimus).

51. Belgian Federal Agency for Medicines and Health Products: International Non-Proprietary Name (INN) Prescription, December 09, 2011.

52. Benet LZ. Relevance of pharmacokinetics in narrow therapeutic index drugs. Transplant Proc 1999; 31: 1642-44.

53. Kesselheim AS, Misono AS, Lee JL, Stedman MR, Brookhart MA, Choudhry NK, Shrank WH. Clinical equivalence of generic and brand-name drugs used in cardiovascular disease. $J \mathrm{Am} \mathrm{Med}$ Assoc 2008; 300: 2514-26. 
54. Verbeeck RK. Bioequivalence, therapeutic equivalence and generic drugs. Acta Clin Belg 2009; 64: 379-83.

55. Gagne JJ, Avorn J, Shrank WH, Schneeweiss S. Refilling and switching of antiepileptic drugs and seizure-related events. Cin Pharmacol Ther 2010; 88:347-53.

56. Moore N, Berdaï D, Bégaud B. Are generic drugs really inferior medicines? Clin Pharmacol Ther 2010; 88: 302-04.

57. HPFB Guidance for Industry: Bioequivalence requirements: critical dose drugs, Ottawa, May 31, 2006(http://www.hc-sc.gc.ca/dhp-

mps/alt_formats/pdf/prodpharma/applic-

demande/guide-ld/bio/critical_dose_critiqueeng.pdf).

58. FDA Guidance for Industry: Bioavailability and Bioequivalence Studies for Orally Administered Drug Products - General Considerations, CDER/FDA, Washington, March 2003 (http://www.fda.gov/downloads/Drugs/GuidanceC omplianceRegulatoryInformation/Guidances/ucm0 70124.pdf).

59. Williams RL. Therapeutic equivalence of generic drugs - Response to National Association of Boards of Pharmacy, Center for Drug Evaluation and Research, FDA, 1997 (www.fda.gov/cder/news.ntiletter.htm).

60. FDA Meeting of the Advisory Committee for Pharmaceutical Science and Clinical Pharmacology; Topic 1: Revising the Bioequivalence (BE) Approaches for Critical Dose Drugs, April 13, 2010 (http:/www.fda.gov/downloads/AdvisoryCommitt ees/CommitteesMeetingMaterials/Drugs/Advisory CommitteeforPharmaceutical-

ScienceandClinicalPharmacology/UCM207955.pd f).

61. FDA ACPS-CP Meeting: Bioequivalence and Quality Standards for Narrow Therapeutic Index Drug Products, July 26, 2011 (http://www.fda.gov/downloads/AdvisoryCommitt ees/CommitteesMeetingMaterials/Drugs/Advisory CommitteeforPharmaceuticalScienceandClinicalP harmacology/UCM263465.pdf).

62. Bialer M, Midha KK. Generic products of antiepileptic drugs: a perspective on bioequivalence and interchangeability. Epilepsia 2010; 51: 941 50 .
63. Amidon GL, Lennernas H, Shah VP, Crison JR. A theoretical basis for a biopharmaceutic drug classification: the correlation of in vitro drug product dissolution and in vivo bioavailability. Pharm Res 1995; 12: 413-20.

64. FDA Guidance for Industry: Waiver of In Vivo Bioavailability and Bioequivalence Studies for Immediate Release Solid Oral Dosage Forms Based on a Biopharmaceutics Classification System, CDER/FDA, Washington, August 2000 (http://www.fda.gov/cder/guidance/index.htm).

65. Blume HH, Schug BS. The biopharmaceutics classification system (BCS): class III drugs better candidates for BA/BE waiver? Eur J Pharm Sci 1999; 9:117-21.

66. Yu LX, Amidon GL, Polli JE, Zhao H, Mehta MU, Conner DP, Shah VP, Lesko LJ, Chen M-L, Lee VHL, Hussain AS. Biopharmaceutics classification system: the scientific basis for biowaiver extensions. Pharm Res 2002; 19: 92125.

67. Polli JE, Yu LX, Cook JA et al. Summary workshop report: Biopharmaceutics Classification System - implementation challenges and extension opportunities. J Pharm Sci 2004; 93: 1375-81.

68. Jantratid E, Prakongpan S, Amidon GL, Dressman JB. Feasibility of biowaiver extension to biopharmaceutics classification system III drug products-cimetidine. Clin Pharmacokinet 2006; 45: 385-99.

69. Wu C-Y, Benet LZ. Predicting drug disposition via application of BCS: transport/absorption/elimination interplay and development of a biopharmaceutics drug disposition classification system. Pharm Res 2005; 22: 11-23.

70. WHO Expert Committee on Specifications for Pharmaceutical Preparations,Annex 8: Proposal to waive in vivo bioequivalence requirements for WHO Model List of Essential Medicines immediate-release, solid oral dosage forms. WHO Technical Report Series No. 937, 2006 (http://www.who.int/medicines/publications/phar mprep/PDF_TRS953_WEB.pdf). 